\title{
It's not just the television: survey analysis of sedentary behaviour in New Zealand young people
}

\author{
Louise S Foley ${ }^{1}$, Ralph Maddison ${ }^{*}$, Yannan Jiang ${ }^{1}$, Timothy Olds $^{2}$ and Kate Ridley ${ }^{3}$
}

\begin{abstract}
Background: Sedentary behaviour has been linked with adverse health outcomes in young people; however, the nature and context of being sedentary is poorly understood. Accurate quantification and description of sedentary behaviour using population-level data is required. The aim of this research was to describe sedentary behaviour among New Zealand (NZ) youth and examine whether sedentary behaviour differs by Body Mass Index (BMI) status in this population.

Methods: A national representative cross-sectional survey of young people aged 5-24 years $(n=2,503)$ was conducted in 2008-2009. Data from this survey, which included subjectively (recall diary; $n=1,309$ ) and objectively (accelerometry; $\mathrm{n}=960$ ) measured sedentary behaviour for participants aged 10-18 years were analysed using survey weighted methods.

Results: Participants self-reported spending on average 521 minutes per day (standard error [SE] 5.29) in total sedentary behaviour, 181 minutes per day (SE 3.91) in screen-based sedentary activities (e.g., television and video games), and 340 minutes per day (SE 5.22) in other non-screen sedentary behaviours (e.g., school, passive transport and self-care). Accelerometer-measured total sedentary behaviour was on average 420 minutes per day (SE 4.26), or 53\% (SE 0.42\%) of monitored time. There were no statistically significant differences in time spent in sedentary behaviour among overweight, obese and healthy/underweight young people.

Conclusions: Both subjective and objective methods indicate that NZ youth spend much of their waking time being sedentary. No relationships were found between sedentary behaviour and BMI status. These findings extend previous research by describing engagement in specific sedentary activities, as well as quantifying the behaviour using an objective method. Differences in what aspects of sedentary behaviour the two methods are capturing are discussed. This research highlights the potential for future interventions to target specific sedentary behaviours or demographic groups.
\end{abstract}

Keywords: Sedentary behaviour, self-report, accelerometry, cross-sectional survey

\section{Background}

Sedentary behaviour in young people has been linked with adverse health outcomes including increased metabolic risk [1] and adiposity [2,3]. It is proposed that changes in the physical and social environment encourage sedentariness across the full spectrum of behavioural contexts, including work, school, home and

\footnotetext{
* Correspondence: r.maddison@ctru.auckland.ac.nz

'Clinical Trials Research Unit, University of Auckland, Private Bag 92019,

Auckland Mail Centre, Auckland 1142, New Zealand

Full list of author information is available at the end of the article
}

transport [4]. Decreasing sedentary time has emerged as an important target for health promotion in conjunction with efforts to promote increased participation in physical activity [5]. However, how and where people are being sedentary is poorly understood, with sedentary behaviour research to date focussing predominantly on leisure-time screen-based pursuits such as television viewing [6]. Accurate quantification and description of sedentary behaviour using population-level data is vital to understand this phenomenon and inform the development of public health interventions.

\section{() Biomed Central}


Sedentary behaviour is not merely the absence of physical activity; rather, it involves purposeful engagement in a large variety of behaviours associated with low energy expenditure [7]. Derived from the Latin sedere ("to sit"), sedentary behaviour encompasses both nonleisure and leisure activities including school work, socialising, passive transport, screen time and nonscreen leisure activities such as arts and crafts and musical pursuits [8]. Sedentary behaviour has been measured both subjectively and objectively [4]. Subjective (selfreport) measures have the advantage of providing detail on the type and context of sedentary behaviour, but are associated with the inherent limitations of all self-report instruments such as social desirability and recall biases [9]. Early research studies involving young people tended to quantify sedentary behaviour using selfreported screen time as a proxy for total sedentary time [6]. However, recent research has utilised use-of-time self-report tools to capture a wider variety of sedentary behaviours $[8,10]$. For example, a national survey of Australian young people aged $9-16$ years $(n=2,200)$ using a use-of-time tool indicated that non-screen sedentary behaviours constituted $60 \%$ of total sedentary time. Moreover, screen time was only moderately correlated with total sedentary time $(r=0.53)$, indicating that screen time may not be appropriate as a proxy for total sedentary time [8].

Sedentary behaviour has also been quantified objectively using accelerometry. Objective measures overcome the biases associated with self-report. They are also associated with low participant burden; however, information regarding the context or nature of the behaviour is not obtained. There is also considerable debate about how sedentary behaviour should be operationalised when using objective measurement devices. Accelerometry has recently been used to quantify sedentary behaviour in several large population-based studies. In a representative cross-sectional survey conducted in the United States (US), Americans aged 6-85 years spent 460 (standard error [SE] 2.4) minutes per day in sedentary behaviour [5]. Adolescents (16-19 years) were one of the most sedentary groups, spending 482 (SE 4.8) minutes per day in sedentary pursuits [5]. A longitudinal study of American adolescent females indicated that sedentary behaviour increased from 461 (standard deviation [SD] 67) to 512 (SD 64) minutes per day between the ages of 11-13 years [11]. Similarly, a United Kingdom (UK)-based longitudinal sample found 11 year old children engaged in 428 (SD 66.4) minutes of sedentary behaviour per day [3]. Because of the relative advantages and disadvantages of subjective and objective measurement of sedentary behaviour, a dual approach using both methods concurrently may be optimal to identify both the types and overall amount of sedentary behaviour young people engage in. Together, this approach has the potential to improve our understanding of when, where and how young people are sedentary.

The overall objective of this research was to comprehensively describe sedentary behaviour among New Zealand (NZ) young people aged $10-18$ years using a dual subjective and objective approach to measurement. Specific aims were to a) quantify self-reported engagement in behavioural sets of sedentary behaviours, broadly classified as screen and non-screen behaviours; b) objectively quantify total sedentary time using accelerometry; c) examine sedentary behaviour outcomes by various demographic sub-groups and d) examine whether sedentary behaviour differs between overweight, obese, and healthy (or underweight) young people. To our knowledge, this is the first study to report concurrently-collected subjective and objective data on sedentary behaviour in a nationally representative sample of children, though this has been done recently in adults [12].

\section{Method}

A national representative cross-sectional survey of $\mathrm{NZ}$ young people aged 5-24 years was conducted between September 2008 and May 2009. The survey was conducted according to the ethical principles outlined in the Declaration of Helsinki and was covered by Statistics New Zealand Tier 1 ethical approval. Written consent was obtained from all participants or their parent, depending on the age of the participant. Sedentary behaviour outcomes for participants aged 10-18 years are reported here.

\section{Study population and design}

A complex survey design involving stratified multi-stage sampling was used, with meshblocks (a defined geographic area) as the primary sampling unit. Within each meshblock, eligible households were identified and asked to participate. One young person was randomly chosen from each eligible household. The overall response rate was $55 \%$. A total of 2,503 young people aged 5-24 years participated in the survey, which consisted of $18.8 \%$ Māori (indigenous population), 9.6\% Pacific, $12.9 \%$ Asian and $71.4 \%$ NZ European. This is representative of the ethnic composition of the general NZ population [13]. A total of 1,315 participants were aged 10-18 years.

\section{Procedure}

Data were collected during a face-to-face home visit and a subsequent telephone interview conducted 7-14 days after the home visit. During the home visit, height and weight were measured and data on demographics and 
self-reported (subjective) physical activity and sedentary behaviour were collected. Accelerometers were then fitted to participants to provide an objective measure of behaviour over a seven-day period. During the telephone interview, additional self-reported data on physical activity and sedentary behaviour were collected.

\section{Measures}

Height was measured to the nearest $0.1 \mathrm{~cm}$ with a stadiometer (Seca, 214, Hamburg, Germany) and weight was measured to the nearest $0.1 \mathrm{~kg}$ with a digital scale (Tanita, UM-070, Illinois, US) according to standard procedures [14]. For both height and weight, two measures were taken. A third measurement was performed if differences of $0.1 \mathrm{~cm}$ and $0.1 \mathrm{~kg}$, respectively were observed between the first and second measurements. The mean of two or the median of three measurements was used in analysis. BMI was calculated from the weight $(\mathrm{kg})$ divided by height $(\mathrm{m})$ squared. International Obesity Task Force [15] classifications of body size were derived.

Self-reported sedentary behaviour was measured using the Multimedia Activity Recall for Children and Adolescents (MARCA) [16]. The MARCA is a computerised use-of-time tool. All daily activities (including sleep) are retrospectively recalled in sequential time segments of five minutes or more for the previous 24 hours. Participants choose from a list of approximately 250 activities. Each activity is linked to an energy cost taken from existing compendia in children [17] and adults [18]. Metabolic Equivalents (METs) [19] are used describe the intensity of activities. The MARCA has been shown to have adequate psychometric properties $[16,20]$. For the current survey up to four days of recall were completed. Participants recalled the two previous days of activity at each of the two data collection periods.

Sedentary behaviour was measured objectively using an Actigraph accelerometer (model AM7164-2.2C). Accelerometers have been validated as an objective measure of physical activity in children [21], adolescents and young adults [22,23], and have also been used to quantify sedentary behaviour $[3,5,11]$. All participants were asked to wear the Actigraph during waking hours for seven consecutive days (including two weekend days). A ten-second epoch was used [21], and data were summed to provide minute-by-minute measurement.

\section{Data treatment MARCA}

MARCA-derived total sedentary time (TST) was defined as waking seated or lying activities at $<3$ METs, as listed in the MARCA compendium. The majority of included activities fell in the range of 2 METs or less; of the 70 activities classified as sedentary, only seven were associated with an energy expenditure of greater than 2 METs.

Screen sedentary time (SST) was defined as the number of waking minutes reported in seated or lying activities at $<3$ METs involving television (sitting or lying), video games, computers or movies and was comprised of five activities. Watching a movie at the cinema was classified under the "movie" category, but watching a movie on the television was classified as "television". Non-screen sedentary time (NSST) was defined as the number of waking minutes reported in seated or lying activities at $<3$ METs not involving a screen, and comprised the remaining 65 activities. NSST was further broken down into six intuitive sets: socialising, school/ work, self-care, passive transport, music and leisure.

TST, SST and NSST derived from available data were divided by the number of days to provide a daily average for each participant. In the total survey population aged 5-24 years, 2,493 (99.6\%) participants provided valid MARCA data, among whom 1,309 (52.5\%) were aged 10-18 years.

\section{Accelerometer}

Accelerometer-derived TST was defined as number of minutes spent in activity eliciting between zero and 100 accelerometer counts per minute [24]. Using published criteria [25] a valid minute was defined as a recorded minute that did not fall into a sequence of $\geq 20$ minutes of zero activity counts. A valid day was defined as a recorded day that had a minimum of 600 valid minutes. Average daily TST was calculated from valid days using valid minutes only. To account for potential differences in valid minutes of accelerometer data between participants, TST was also expressed as a percentage of valid minutes recorded for each participant. In the total survey population aged 5-24 years, 1,812 (72.4\%) participants provided valid accelerometer data, among whom 960 (53.0\%) were aged 10-18 years.

\section{Statistical analysis}

Statistical analyses were performed using statistical software SAS version 9.2 (SAS Institute Inc, Cary NC) and $\mathrm{R}$ version 2.12.0 (R Foundations for Statistical Computing). Survey weights appropriate for stratified multistage samplings were calculated to estimate the population means and SEs using the jack-knife replication method. For all variables, data are presented descriptively. Demographic sub-groups were defined including gender, age, deprivation, area (rural vs. urban), ethnicity and overweight status. Deprivation level was defined according to the $2006 \mathrm{NZ}$ Deprivation Index [26] (I = least deprived, $\mathrm{V}=$ most deprived). Ethnicity was evaluated using total response which showed the counts of all responses given for each ethnic group $[27,28]$. 
International Obesity Task Force criteria were used to define overweight status from BMI [15,29].

Weighted regression analysis was conducted to examine whether time spent in various sedentary behaviours differed among overweight, obese, and healthy/underweight children (i.e. BMI status), adjusting for their age in years, gender, prioritised ethnicity, and NZ Deprivation Index. A total of 1,300 participants aged 10-18 years who provided valid BMI data were included in this analysis. MARCA derived TST, SST and NSST, as well as accelerometer-derived TST, were considered as the outcomes of interest. BMI status was used as the independent predictor (underweight and healthy weight were combined due to very small numbers).

\section{Results}

\section{MARCA}

Daily time (minutes) spent in TST, SST and NSST for participants aged 10-18 years is presented in Table 1. Overall, young people reported engaging in 521 minutes (SE 5.29) of sedentary behaviour per day, comprised of 181 (SE 3.91) minutes of screen-based activities (Figure 1) and 340 (SE 5.22) minutes of non-screen activities (Figure 2).

For SST, males reported higher use of sedentary screen technologies than females (197 minutes per day [SE 5.78] versus 162 minutes per day [SE 5.01], respectively). Older participants reported greater SST than younger participants (194 minutes per day [SE 7.63] in 15-18 year olds versus 174 minutes per day [SE 4.45] in 10-14 year olds). Asian participants reported higher use of screen-based technologies (207 minutes per day [SE 12.62]) than any other ethnic group. Daily time (minutes) spent in categories of SST is presented in Table 2. Television watching was the predominant screen-based activity, with reported use of 132 minutes per day (SE $3.25)$ in the whole sample.

For NSST, females reported higher engagement in non-screen based sedentary activities than males (364

Table 1 Daily engagement in sedentary behaviour types derived by self-report (MARCA)

\begin{tabular}{|c|c|c|c|c|c|c|c|c|c|}
\hline \multirow[t]{2}{*}{ Variable } & \multicolumn{3}{|c|}{ Total sedentary time $(\mathrm{min})$} & \multicolumn{3}{|c|}{ Screen sedentary time (min) } & \multicolumn{3}{|c|}{ Non-screen sedentary time (min) } \\
\hline & $n^{1}$ & Mean $^{2}$ & $S E^{3}$ & $n^{1}$ & Mean $^{2}$ & $S E^{3}$ & $n^{1}$ & Mean $^{2}$ & $S E^{3}$ \\
\hline All & 1,309 & 521.1 & 5.29 & 1,309 & 181.1 & 3.91 & 1,309 & 340.0 & 5.22 \\
\hline \multicolumn{10}{|l|}{ Gender } \\
\hline female & 606 & 526.4 & 7.64 & 606 & 162.3 & 5.01 & 606 & 364.1 & 7.62 \\
\hline male & 703 & 516.6 & 7.30 & 703 & 196.9 & 5.78 & 703 & 319.7 & 6.75 \\
\hline \multicolumn{10}{|l|}{ Age group } \\
\hline $10-14$ years & 825 & 508.0 & 6.43 & 825 & 174.0 & 4.45 & 825 & 334.0 & 5.68 \\
\hline $15-18$ years & 484 & 544.4 & 9.14 & 484 & 193.6 & 7.63 & 484 & 350.8 & 9.69 \\
\hline \multicolumn{10}{|l|}{ Deprivation } \\
\hline 1 & 275 & 541.9 & 13.89 & 275 & 168.5 & 6.29 & 275 & 373.4 & 13.05 \\
\hline$\|$ & 264 & 528.6 & 9.50 & 264 & 191.4 & 9.90 & 264 & 337.2 & 8.69 \\
\hline III & 277 & 528.1 & 11.35 & 277 & 179.1 & 8.11 & 277 & 349.0 & 10.91 \\
\hline IV & 216 & 518.7 & 10.35 & 216 & 178.0 & 9.63 & 216 & 340.7 & 11.22 \\
\hline V & 272 & 491.8 & 12.54 & 272 & 188.5 & 9.75 & 272 & 303.3 & 12.57 \\
\hline \multicolumn{10}{|l|}{ Area } \\
\hline rural & 209 & 520.1 & 10.81 & 209 & 177.3 & 9.17 & 209 & 342.8 & 10.16 \\
\hline urban & 1,100 & 521.2 & 5.80 & 1,100 & 181.5 & 4.26 & 1,100 & 339.7 & 5.74 \\
\hline \multicolumn{10}{|l|}{ Ethnicity } \\
\hline Māori & 248 & 474.1 & 12.55 & 248 & 174.1 & 10.15 & 248 & 300.0 & 12.14 \\
\hline Pacific & 131 & 516.4 & 13.39 & 131 & 182.5 & 13.80 & 131 & 333.9 & 13.79 \\
\hline Asian & 174 & 600.5 & 10.30 & 174 & 206.8 & 12.62 & 174 & 393.7 & 11.23 \\
\hline NZ Euro/Other & 923 & 524.0 & 6.50 & 923 & 181.0 & 4.48 & 923 & 343.0 & 6.73 \\
\hline \multicolumn{10}{|l|}{ OW status } \\
\hline underweight & 61 & 532.7 & 26.88 & 61 & 201.1 & 16.16 & 61 & 331.6 & 24.53 \\
\hline healthy & 788 & 523.2 & 6.62 & 788 & 179.4 & 5.57 & 788 & 343.9 & 6.47 \\
\hline overweight & 303 & 522.7 & 9.99 & 303 & 176.6 & 7.81 & 303 & 346.1 & 10.87 \\
\hline obese & 147 & 495.8 & 15.85 & 147 & 189.7 & 11.03 & 147 & 306.1 & 14.60 \\
\hline
\end{tabular}

${ }^{1}$ Number of participants in each category

${ }^{2}$ Weighted mean estimate

${ }^{3}$ Standard error of the mean

MARCA $=$ Multimedia Activity Recall for Children and Adolescents 


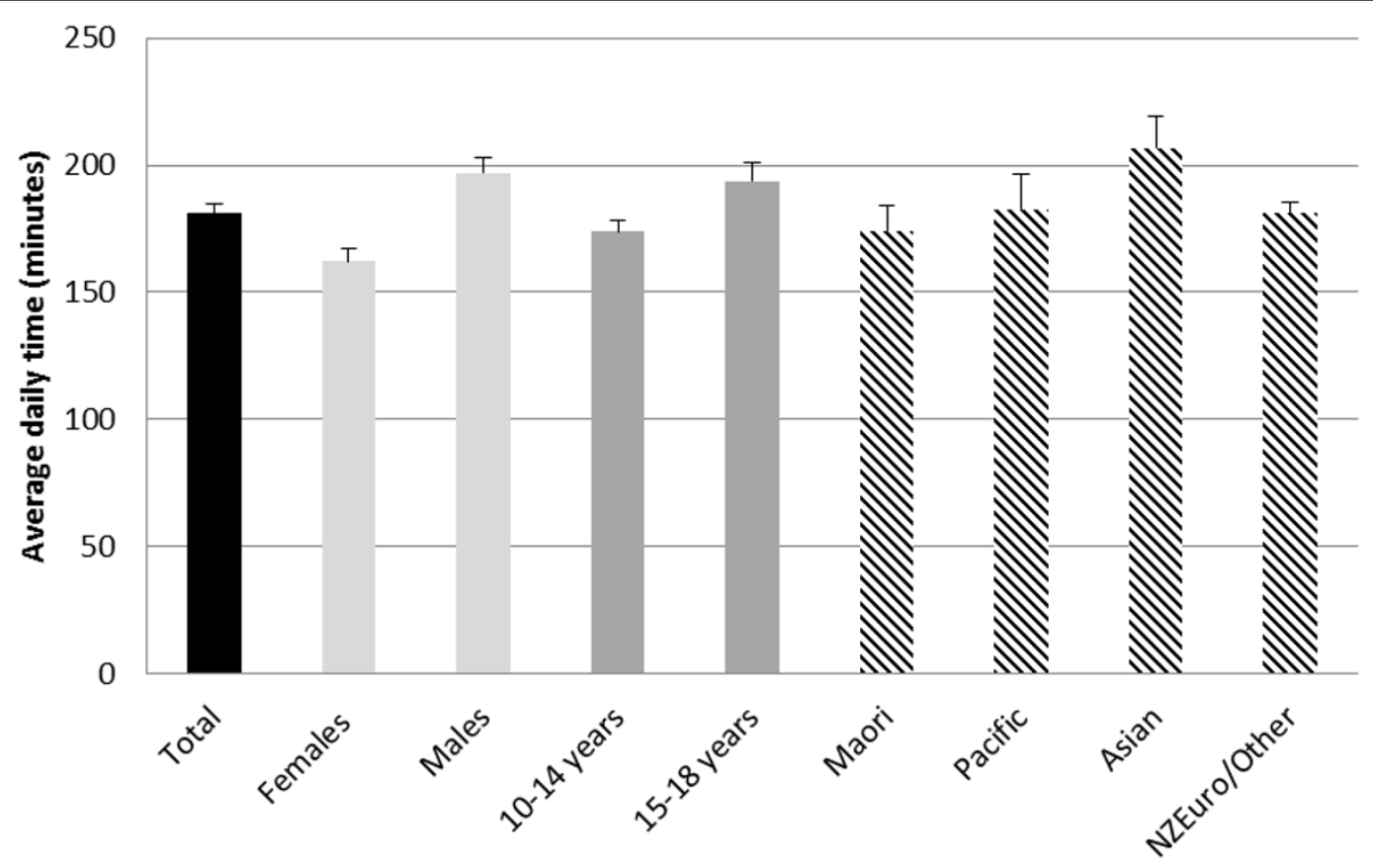

Figure 1 Self-reported (MARCA) screen sedentary time by population sub-group.

minutes per day [SE 7.62] versus 320 minutes per day [SE 6.75] respectively). Those of the lowest deprivation level reported the greatest amount of NSST (373 minutes per day [SE 13.05]), compared with those in the highest deprivation level who reported the least (303 minutes per day [SE 12.57]). Asian participants reported more NSST (394 minutes per day [SE 11.23]) than any other ethnic group. Daily time (minutes) spent in

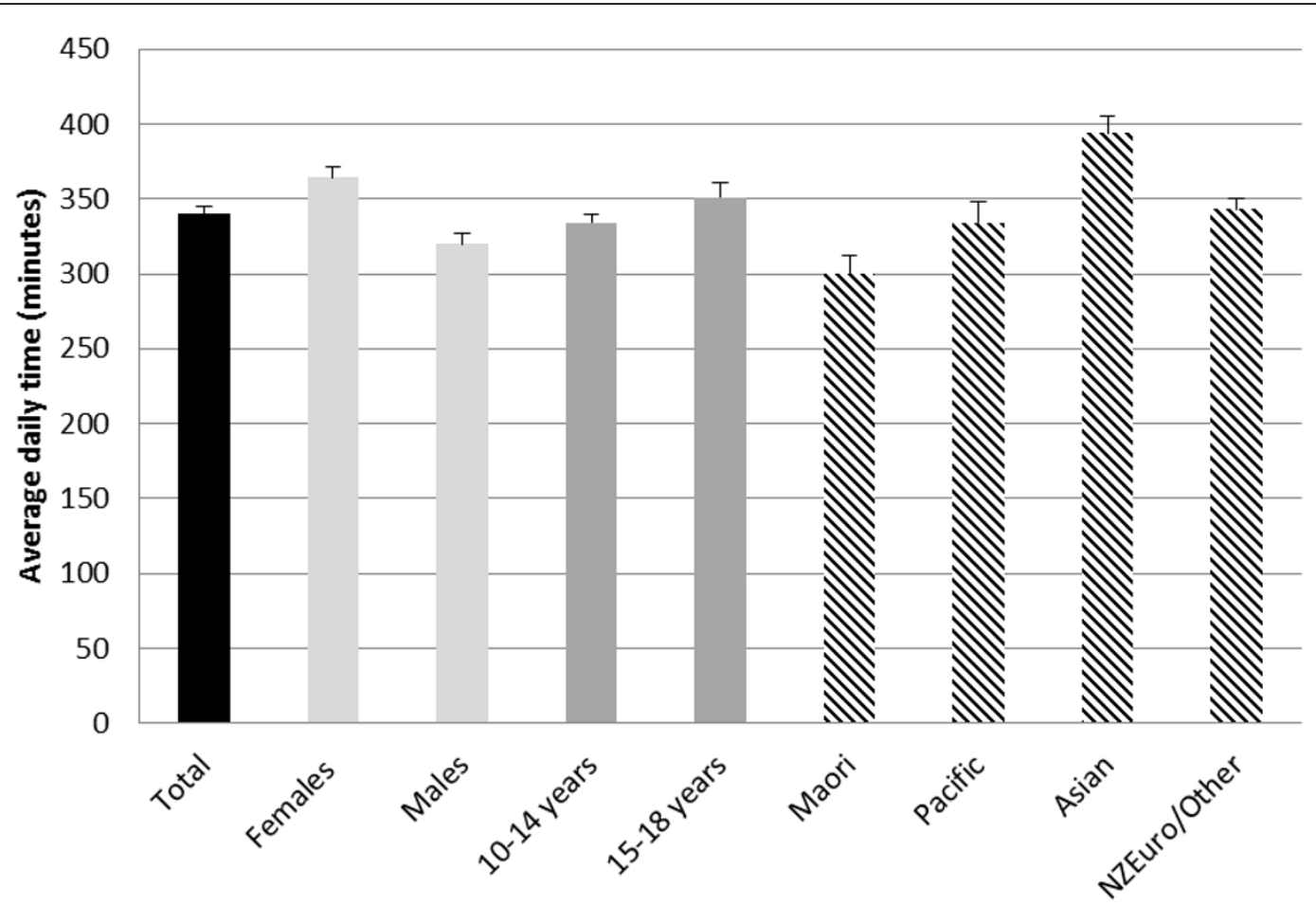

Figure 2 Self-reported (MARCA) non-screen sedentary time by population sub-group 
Table 2 Daily engagement in categories of screen sedentary time derived by self-report (MARCA)

\begin{tabular}{|c|c|c|c|c|c|c|c|c|c|}
\hline \multirow[t]{2}{*}{ Variable } & \multicolumn{3}{|c|}{ Television (min) } & \multicolumn{3}{|c|}{ Computer/video game (min) } & \multicolumn{3}{|c|}{ Movie (min) } \\
\hline & $n^{1}$ & Mean $^{2}$ & $S E^{3}$ & $n^{1}$ & Mean $^{2}$ & $S E^{3}$ & $n^{1}$ & Mean $^{2}$ & $S E^{3}$ \\
\hline$\overline{\text { All }}$ & 1,309 & 131.9 & 3.25 & 1,309 & 46.7 & 2.32 & 1,309 & 2.5 & 0.46 \\
\hline \multicolumn{10}{|l|}{ Gender } \\
\hline female & 606 & 126.2 & 4.30 & 606 & 33.0 & 2.67 & 606 & 3.1 & 0.78 \\
\hline male & 703 & 136.8 & 4.81 & 703 & 58.3 & 3.51 & 703 & 1.9 & 0.54 \\
\hline \multicolumn{10}{|l|}{ Age group } \\
\hline $10-14$ years & 825 & 131.8 & 3.83 & 825 & 40.4 & 2.48 & 825 & 1.8 & 0.51 \\
\hline $15-18$ years & 484 & 132.2 & 5.88 & 484 & 57.8 & 4.56 & 484 & 3.6 & 0.90 \\
\hline \multicolumn{10}{|l|}{ Deprivation } \\
\hline I & 275 & 116.1 & 5.09 & 275 & 48.4 & 4.78 & 275 & 3.9 & 1.48 \\
\hline$\|$ & 264 & 134.6 & 8.99 & 264 & 53.1 & 5.32 & 264 & 3.7 & 1.29 \\
\hline III & 277 & 129.0 & 5.96 & 277 & 47.6 & 4.20 & 277 & 2.4 & 0.96 \\
\hline IV & 216 & 127.6 & 7.40 & 216 & 49.2 & 7.02 & 216 & 1.2 & 0.59 \\
\hline V & 272 & 151.1 & 8.28 & 272 & 36.1 & 4.40 & 272 & 1.2 & 0.51 \\
\hline \multicolumn{10}{|l|}{ Area } \\
\hline rural & 209 & 137.4 & 6.94 & 209 & 38.6 & 5.07 & 209 & 1.3 & 0.77 \\
\hline urban & 1,100 & 131.2 & 3.56 & 1,100 & 47.7 & 2.53 & 1,100 & 2.6 & 0.51 \\
\hline \multicolumn{10}{|l|}{ Ethnicity } \\
\hline Māori & 248 & 139.1 & 8.51 & 248 & 34.4 & 4.30 & 248 & 0.7 & 0.30 \\
\hline Pacific & 131 & 149.8 & 12.76 & 131 & 29.5 & 4.41 & 131 & 3.1 & 1.50 \\
\hline Asian & 174 & 132.0 & 9.77 & 174 & 72.6 & 7.92 & 174 & 2.3 & 1.28 \\
\hline NZ Euro/Other & 923 & 131.1 & 3.75 & 923 & 47.2 & 2.77 & 923 & 2.8 & 0.58 \\
\hline \multicolumn{10}{|l|}{ OW status } \\
\hline underweight & 61 & 136.4 & 11.95 & 61 & 61.5 & 13.70 & 61 & 3.1 & 2.00 \\
\hline healthy & 788 & 125.9 & 4.51 & 788 & 50.6 & 3.19 & 788 & 2.9 & 0.67 \\
\hline overweight & 303 & 135.7 & 6.65 & 303 & 39.5 & 4.28 & 303 & 1.4 & 0.47 \\
\hline obese & 147 & 153.1 & 11.25 & 147 & 34.4 & 4.32 & 147 & 2.3 & 1.40 \\
\hline
\end{tabular}

${ }^{1}$ Number of survey participants in each category

${ }^{2}$ Weighted estimate of the mean

${ }^{3}$ Standard error of the estimate calculated using the replicated weights

MARCA - Multimedia Activity Recall for Children and Adolescents

categories of NSST is presented in Table 3. Overall, school/work activities were the most frequently reported (145 minutes per day; SE 4.55), followed by socialising (45 minutes per day; SE 2.07), passive transport (42 minutes per day; SE 1.47), sedentary leisure activities (39 minutes per day; SE 2.01) and music (11 minutes per day; SE 0.97).

\section{Accelerometer}

Daily time (minutes) spent in TST is presented in Table 4 and Figure 3. Young people aged 10-18 years spent 420 minutes per day (SE 4.26) engaged in sedentary behaviour, or $53 \%$ (SE $0.42 \%$ ) of monitored time. TST was similar for females and males. However, there were differences between age groups; the younger age group (10-14 years) spent 400 minutes per day (SE 4.95) in sedentary behaviour (50\% [SE $0.48 \%$ ] of monitored time), compared with
460 minutes per day (SE 7.64) (58\% [SE 0.74\%] of monitored time) in the older age group (15-18 years). Participants of Asian ethnicity were the most sedentary of all ethnic groups, spending an average of 472 minutes per day (SE 11.49) engaged in sedentary behaviour (59\% [SE $1.10 \%$ ] of monitored time), compared with Māori who were the least sedentary, with an average of 386 minutes per day (SE 9.29) (49\% [SE 0.88\%] of monitored time).

\section{Relationship between sedentary behaviour and BMI status}

The adjusted survey regression analysis indicated no statistically significant differences between underweight/ healthy weight, overweight and obese participants for time spent in sedentary behaviour.

For MARCA-derived SST, obese participants engaged in 14.5 minutes more per day than underweight/healthy 
Table 3 Daily engagement in categories of non-screen sedentary time derived by self-report (MARCA)

\begin{tabular}{|c|c|c|c|c|c|c|c|c|c|c|c|c|c|c|c|}
\hline \multirow[t]{2}{*}{ Variable } & \multicolumn{3}{|c|}{ Socialising (min) } & \multicolumn{3}{|c|}{ School/work (min) } & \multicolumn{3}{|c|}{ Passive transport (min) } & \multicolumn{3}{|c|}{ Music (min) } & \multicolumn{3}{|c|}{ Leisure (min) } \\
\hline & $n^{1}$ & Mean $^{2}$ & $S E^{3}$ & $n^{\prime}$ & Mean $^{2}$ & $S E^{3}$ & $n^{\prime}$ & Mean $^{2}$ & $S E^{3}$ & $n^{1}$ & Mean $^{2}$ & $S E^{3}$ & $n^{7}$ & $\mathrm{Mean}^{2}$ & $S E^{3}$ \\
\hline All & 1,309 & 45.1 & 2.07 & 1,309 & 145.2 & 4.55 & 1,309 & 42.4 & 1.47 & 1,309 & 10.8 & 0.97 & 1,309 & 38.8 & 2.01 \\
\hline \multicolumn{16}{|l|}{ Gender } \\
\hline female & 606 & 55.3 & 2.90 & 606 & 147.5 & 7.10 & 606 & 45.2 & 2.32 & 606 & 11.9 & 1.29 & 606 & 40.9 & 2.76 \\
\hline male & 703 & 36.5 & 2.32 & 703 & 143.3 & 6.06 & 703 & 40.0 & 1.71 & 703 & 9.9 & 1.38 & 703 & 37.1 & 2.57 \\
\hline \multicolumn{16}{|l|}{ Age group } \\
\hline $10-14$ years & 825 & 36.1 & 2.03 & 825 & 153.5 & 4.34 & 825 & 38.3 & 1.41 & 825 & 9.6 & 0.87 & 825 & 41.5 & 2.32 \\
\hline $15-18$ years & 484 & 61.1 & 3.78 & 484 & 130.5 & 9.75 & 484 & 49.7 & 3.06 & 484 & 12.9 & 2.19 & 484 & 34.1 & 3.25 \\
\hline \multicolumn{16}{|l|}{ Deprivation } \\
\hline 1 & 275 & 45.0 & 3.38 & 275 & 160.6 & 9.66 & 275 & 51.1 & 2.95 & 275 & 10.1 & 1.57 & 275 & 48.3 & 4.43 \\
\hline$\|$ & 264 & 37.0 & 3.33 & 264 & 146.3 & 7.56 & 264 & 45.5 & 2.79 & 264 & 8.9 & 1.61 & 264 & 41.3 & 4.50 \\
\hline III & 277 & 42.0 & 4.27 & 277 & 156.8 & 11.86 & 277 & 41.8 & 2.72 & 277 & 10.8 & 1.67 & 277 & 38.5 & 3.16 \\
\hline IV & 216 & 56.1 & 6.99 & 216 & 128.3 & 10.73 & 216 & 40.3 & 4.92 & 216 & 14.2 & 2.59 & 216 & 39.8 & 6.40 \\
\hline V & 272 & 45.0 & 4.18 & 272 & 135.1 & 11.09 & 272 & 33.9 & 2.86 & 272 & 10.2 & 2.89 & 272 & 27.4 & 3.64 \\
\hline \multicolumn{16}{|l|}{ Area } \\
\hline rural & 209 & 40.5 & 3.73 & 209 & 144.2 & 8.83 & 209 & 56.5 & 3.08 & 209 & 9.6 & 2.17 & 209 & 37.8 & 3.93 \\
\hline urban & 1,100 & 45.7 & 2.28 & 1,100 & 145.4 & 5.01 & 1,100 & 40.6 & 1.59 & 1,100 & 11.0 & 1.06 & 1,100 & 39.0 & 2.21 \\
\hline \multicolumn{16}{|l|}{ Ethnicity } \\
\hline Māori & 248 & 45.2 & 4.34 & 248 & 126.3 & 11.61 & 248 & 36.7 & 2.94 & 248 & 13.5 & 3.15 & 248 & 31.8 & 5.81 \\
\hline Pacific & 131 & 57.4 & 6.85 & 131 & 139.4 & 12.24 & 131 & 38.2 & 3.17 & 131 & 11.1 & 2.13 & 131 & 37.4 & 6.16 \\
\hline Asian & 174 & 41.4 & 4.36 & 174 & 188.7 & 10.41 & 174 & 39.4 & 2.75 & 174 & 8.3 & 1.40 & 174 & 43.3 & 4.59 \\
\hline NZ Euro/Other & 923 & 44.4 & 2.55 & 923 & 141.1 & 5.61 & 923 & 45.2 & 1.86 & 923 & 10.2 & 0.92 & 923 & 41.8 & 2.30 \\
\hline \multicolumn{16}{|l|}{ OW status } \\
\hline underweight & 61 & 39.3 & 8.93 & 61 & 134.9 & 18.81 & 61 & 36.2 & 5.41 & 61 & 6.7 & 2.90 & 61 & 47.7 & 12.43 \\
\hline healthy & 788 & 45.8 & 2.82 & 788 & 142.7 & 5.47 & 788 & 43.9 & 1.95 & 788 & 12.2 & 1.43 & 788 & 38.1 & 2.20 \\
\hline overweight & 303 & 45.8 & 3.83 & 303 & 155.9 & 12.66 & 303 & 42.9 & 2.53 & 303 & 8.6 & 1.55 & 303 & 44.6 & 4.59 \\
\hline obese & 147 & 42.9 & 5.09 & 147 & 135.2 & 10.17 & 147 & 35.2 & 4.01 & 147 & 9.6 & 1.69 & 147 & 28.7 & 3.91 \\
\hline
\end{tabular}

${ }^{1}$ Number of survey participants in each category

${ }^{2}$ Weighted estimate of the mean

${ }^{3}$ Standard error of the estimate calculated using the replicated weights

MARCA - Multimedia Activity Recall for Children and Adolescents

weight participants (95\% CI -13.4 to $42.4 ; \mathrm{p}=0.31$ ). For MARCA-derived NSST, overweight participants engaged in 8.1 minutes more per day than underweight/healthy weight participants ( $95 \% \mathrm{CI}-16.5$ to $32.7 ; \mathrm{p}=0.52$ ) and obese participants engaged in 15.0 minutes less per day than underweight/healthy weight participants $(95 \% \mathrm{CI}$ -46.3 to $16.3 ; \mathrm{p}=0.35$ ).

\section{Discussion}

The aim of this research was to describe sedentary behaviours among NZ young people aged 10-18 years. Results from both data sources indicate this group spend much of their waking time in sedentary pursuits, consistent with reports from other developed countries $[3,5,30,31]$.

SST (most commonly television watching) has been reduced successfully in previous interventions, with concurrent improvements in weight indices [32]. SST is thought to have adverse effects on body composition via displacement of physical activity, reduction of metabolic rate, exposure to food advertising and promoting snacking [32], and adverse psychosocial effects through exposure to violence and adult content [33]. NZ young people used screen technologies for 181 (SE 3.91) minutes per day on average, somewhat lower than an Australian estimate of 230 (SD 114) minutes per day in adolescents aged 9-16 years in a survey that also used the MARCA [8]. However, these estimates are still markedly lower than recent data from the US, which found that 8-18 year olds reported spending 324 (no SD reported) minutes per day in SST as it is defined in this study [34].

Though SST is an important target for intervention, it should be noted that the daily time reported in NSST 
was nearly twice that of screen-based activities. NZ young people engaged in non-screen sedentary behaviour for 340 (SE 5.22) minutes per day on average. This estimate is very similar to a MARCA-derived NSST estimate of 345 (SD 105) minutes per day in Australian adolescents aged 9-16 [8]. Some non-screen activities such as school may be considered less discretionary and others such as reading may be considered more socially desirable than screen time. However, it is still possible to intervene to break up extended periods of sedentary time and increase energy expended. Modification of the school environment to allow standing lessons and opportunities for physical activity has been shown to significantly increase movement in children [35]. In adults, breaking up sedentary time has been associated with favourable body composition and metabolic risk, independent of total sedentary time and physical activity level [36]. It may also be appropriate to encourage displacement of sedentary socialising and transport with more active alternatives, as each accounted for approximately 45 minutes of daily activity in this sample.

The accelerometry data indicated that young people spent 420 minutes (SE 4.26) in sedentary behaviour per day. This estimate is broadly consistent with surveys from other developed countries conducted in adolescents using the 100 count per minute cut-point. Estimates for total sedentary time from other countries include 452 (SE 6.0) minutes per day in 12-15 year olds and 482 (SE 4.8) minutes per day in 16-19 year olds in a representative US sample [5], 512 (SD 54) minutes per day in a longitudinal US sample aged 13 years [11], 428 (SD 66.4) minutes per day in a longitudinal UK sample aged 11 years [3] and 496 (SD 80.6) and 471 (SD 84.3) minutes per day in a Spanish boys and girls, respectively, aged 13-16 years [30]. Consistent with the welldocumented decline in physical activity from childhood through adolescence to adulthood [37], the accelerometry data also indicated an increase in sedentary behaviour with age. Children aged 10-14 years engaged in 60 minutes less daily sedentary behaviour than those aged $15-18$ years (400 [SE 4.95] minutes or $50 \%$ of monitored time versus 460 [SE 7.64] minutes or $58 \%$ of monitored time, respectively). This indicates a disturbing trend in health-related behaviour across adolescence. However, contrary with reports from other countries [2,3], this study found no differences in engagement in total or specific types of sedentary behaviour between overweight and obese participants and their healthy or underweight counterparts.

The results of this study suggest that the most appropriate targets for intervening to reduce screen time in NZ are males, those in their late teens, and those of Asian ethnicity. Although television watching was the predominant screen behaviour, computer and video games were used for approximately 45 minutes per day. Moreover, interventions to reduce NSST in NZ young people may be more appropriate for females, who report greater levels of NSST than males, and those of Asian ethnicity who reported high levels of NSST as well as high levels of SST. For Asian participants, the MARCA data indicated a greater engagement in computer- and school-based sedentary behaviour. This likely reflects an academic rather than a leisure orientation in sedentary behaviours. Culturally-appropriate interventions should consider the nature of sedentary behaviour in this ethnic group.

The specific strengths of the study are the use of a large, representative sample of NZ young people and the use of the survey methodology for analysis. To the best of our knowledge, this is the first time that complementary methodologies (accelerometry and self-report) have been used to describe sedentary behaviour in a sample of children this large. In adults, it is recommended that population-based monitoring of sedentary time include both self-reported and device-based measurement [12]. However, several limitations warrant discussion. Firstly, this was a cross-sectional study, which does not allow for inferences of causality, or capture change across time. Secondly, there was a $45 \%$ non-participation rate and it is possible that non-participants differed from participants, though the study population was nationally representative by ethnicity, age and geography. During the original survey parents were approached to recruit their child, thus selection bias was based on parent rather than the young person, which might dilute this effect.

Data from a subset of participants aged 10-18 years were analysed rather than the total sample. Exclusion of those aged 19-24 years was considered the most appropriate approach to provide meaningful comparisons of sedentary behaviour amongst young people at a similar stage of development (pre-adolescent or adolescent), as this older age group are often at a transitional stage of life in which they move from school into sedentary, office-based occupations. Exclusion of those aged 5-9 years was for pragmatic reasons because of differences in the way data was collected. For this younger age group, a parent gave a proxy report of activities the child performed whilst being directly supervised by the parent. Therefore, all of the time spent at school was coded as "school", and no further information was provided about sedentary behaviour or physical activity during this time. This was not comparable with older participations who recalled a full 24 hours of activity. Finally, the use of a 10-18 year old age group allows for comparison with other international data sets. 
Table 4 Daily engagement in sedentary behaviour derived by accelerometry

\begin{tabular}{|c|c|c|c|c|c|c|c|c|c|}
\hline \multirow[t]{2}{*}{ Variable } & \multicolumn{3}{|c|}{$\begin{array}{l}\text { Daily valid accelerometer time } \\
\text { (min) }\end{array}$} & \multicolumn{3}{|c|}{$\begin{array}{l}\text { Total sedentary time }(\mathrm{min}) \\
(0 \leq \text { counts per minute } \leq \\
100)\end{array}$} & \multicolumn{3}{|c|}{$\begin{array}{l}\text { Percentage of daily valid minutes spent in sedentary } \\
\text { behaviour }\end{array}$} \\
\hline & $n^{1}$ & Mean $^{2}$ & $S E^{3}$ & $n^{1}$ & Mean $^{2}$ & $S E^{3}$ & $n^{1}$ & Mean $^{2}$ & $S E^{3}$ \\
\hline All & 960 & 789.6 & 4.71 & 960 & 419.5 & 4.26 & 960 & $52.9 \%$ & $0.42 \%$ \\
\hline \multicolumn{10}{|l|}{ Gender } \\
\hline female & 440 & 778.0 & 5.52 & 440 & 421.3 & 5.98 & 440 & $54.0 \%$ & $0.63 \%$ \\
\hline male & 520 & 799.2 & 7.17 & 520 & 418.0 & 6.03 & 520 & $52.0 \%$ & $0.57 \%$ \\
\hline \multicolumn{10}{|l|}{ Age group } \\
\hline $10-14$ years & 646 & 789.5 & 5.70 & 646 & 399.8 & 4.95 & 646 & $50.4 \%$ & $0.48 \%$ \\
\hline $15-18$ years & 314 & 789.7 & 7.54 & 314 & 459.7 & 7.64 & 314 & $58.1 \%$ & $0.74 \%$ \\
\hline \multicolumn{10}{|l|}{ Deprivation } \\
\hline I & 211 & 770.0 & 7.29 & 211 & 421.3 & 7.83 & 211 & $54.6 \%$ & $0.91 \%$ \\
\hline$\|$ & 204 & 790.5 & 10.04 & 204 & 428.1 & 8.56 & 204 & $54.1 \%$ & $0.84 \%$ \\
\hline III & 210 & 796.2 & 7.59 & 210 & 424.1 & 8.85 & 210 & $53.0 \%$ & $0.87 \%$ \\
\hline IV & 152 & 787.5 & 10.18 & 152 & 402.6 & 12.13 & 152 & $50.7 \%$ & $1.09 \%$ \\
\hline V & 180 & 805.6 & 15.01 & 180 & 418.9 & 11.11 & 180 & $51.7 \%$ & $0.89 \%$ \\
\hline \multicolumn{10}{|l|}{ Area } \\
\hline rural & 152 & 802.0 & 8.87 & 152 & 420.8 & 7.26 & 152 & $52.3 \%$ & $0.88 \%$ \\
\hline urban & 808 & 787.9 & 5.20 & 808 & 419.3 & 4.73 & 808 & $53.0 \%$ & $0.46 \%$ \\
\hline \multicolumn{10}{|l|}{ Ethnicity } \\
\hline Māori & 166 & 789.2 & 10.27 & 166 & 386.1 & 9.29 & 166 & $48.6 \%$ & $0.88 \%$ \\
\hline Pacific & 82 & 826.6 & 21.96 & 82 & 426.8 & 17.78 & 82 & $51.1 \%$ & $1.42 \%$ \\
\hline Asian & 118 & 799.2 & 11.40 & 118 & 471.7 & 11.49 & 118 & $58.9 \%$ & $1.10 \%$ \\
\hline NZ Euro/Other & 717 & 781.0 & 4.53 & 717 & 414.8 & 4.29 & 717 & $53.0 \%$ & $0.46 \%$ \\
\hline \multicolumn{10}{|l|}{ OW status } \\
\hline underweight & 52 & 756.9 & 18.32 & 52 & 424.0 & 15.04 & 52 & $56.0 \%$ & $1.46 \%$ \\
\hline healthy & 591 & 790.4 & 5.62 & 591 & 423.4 & 5.63 & 591 & $53.3 \%$ & $0.54 \%$ \\
\hline overweight & 215 & 788.2 & 8.50 & 215 & 412.9 & 7.42 & 215 & $52.3 \%$ & $0.78 \%$ \\
\hline obese & 99 & 804.1 & 17.19 & 99 & 408.6 & 15.20 & 99 & $50.6 \%$ & $1.26 \%$ \\
\hline
\end{tabular}

${ }^{1}$ Number of survey participants in each category

${ }^{2}$ Weighted estimate of the mean

${ }^{3}$ Standard error of the estimate calculated using the replicated weight

The tools used to capture sedentary behaviour are each associated with their own limitations. As discussed, the MARCA data may be affected by the biases associated with self-report, though the psychometric properties have been shown to be sound, and there was a very high proportion of valid data. For accelerometry, it is important to note that no current consensus exists on the most appropriate way to define sedentary behaviour, with published definitions ranging from 100-1100 activity counts per minute (cpm) [38]. Furthermore, estimates relied on participant compliance with wearing the accelerometer during waking hours. When compared to the MARCA, a smaller proportion of participants provided valid data ( $99.6 \%$ vs. $72.4 \%$ ).

Further discussion of issues associated with the measurement of sedentary behaviour via self-report and accelerometry is warranted. Sedentary behaviour is an emerging field of research in public health, but there remains little consensus regarding the optimal way of defining and measuring it. In this survey, the MARCA and accelerometry captured distinct aspects of sedentary behaviour. A seated or lying body posture was the key criterion for defining sedentary behaviour according to the MARCA, with a secondary MET threshold applied. For example, "watching TV sitting - 1.2 METs" was classified as a sedentary behaviour, whereas "playing the drums - 4.0 METs" was not, even though this was a seated behaviour. Conversely, accelerometry defines sedentary behaviour as low movement and does not provide any information on body posture. Standing still can elicit low accelerometer counts and therefore accelerometer-derived sedentary time will 


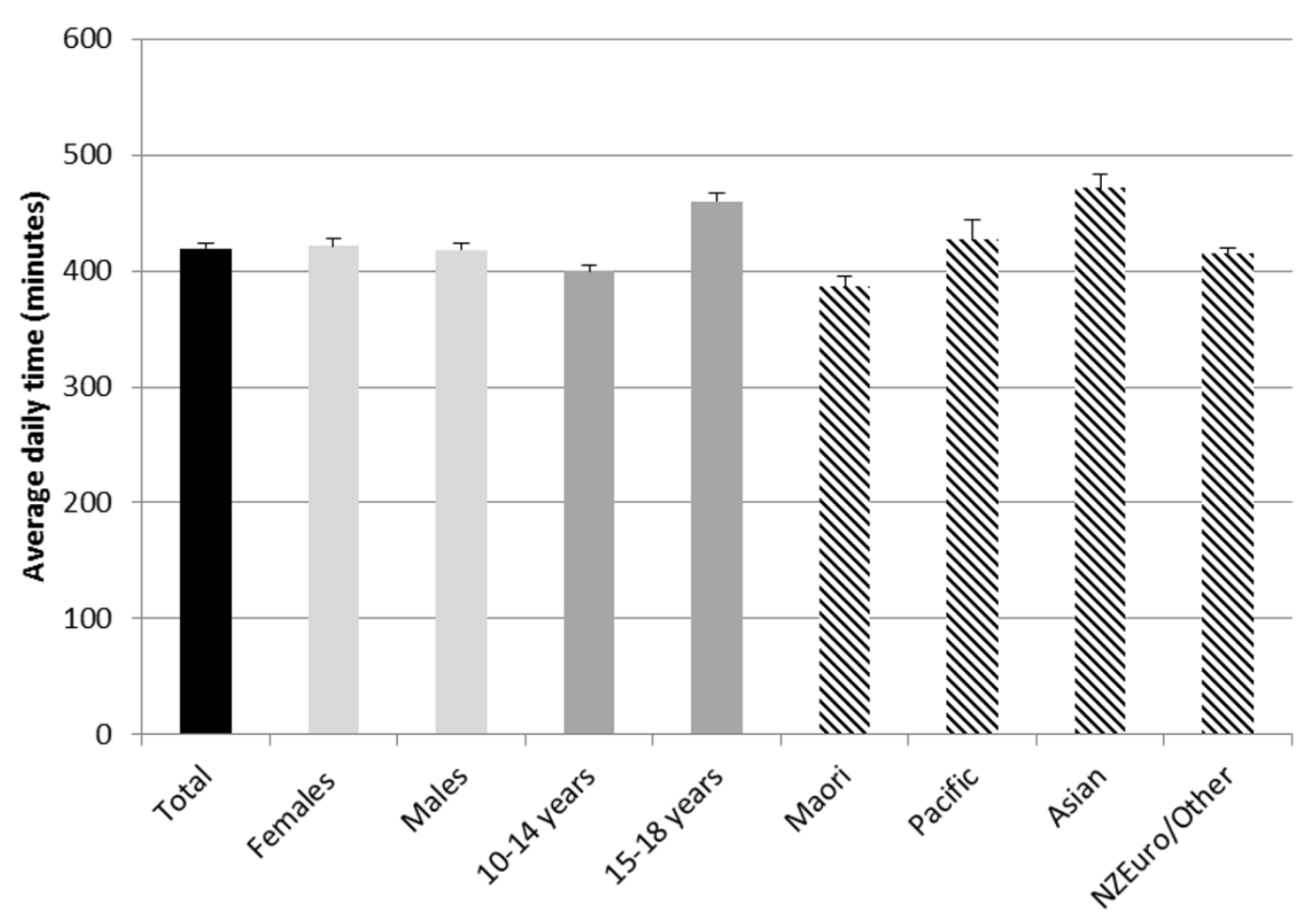

Figure 3 Objectively measured (accelerometer) total sedentary time by population sub-group.

likely include a mixture of time spent both sitting and standing.

For total sedentary behaviour, estimates from the MARCA (521 [SE 5.29] minutes per day) were higher than those from accelerometry (420 [SE 4.26] minutes per day). The 100 minute discrepancy between methods raises the question as to which estimate best represents the "true" value. Differences in the operationalisation of sedentary behaviour, as well as differences in what the tools actually measure, most likely accounts for the discrepancy in outputs. Because of their objectivity, accelerometers have inherent appeal, but are not a criterion method for assessing sedentary behaviour. A new class of devices based on inclinometry (e.g., the activPAL) show promise for the objective assessment of body posture [39]. Though it relies on self-report, the MARCA has potential to provide additional information on what individuals do when they are sedentary. It is likely that the "true" value lies somewhere between the two estimates. Though the absolute value of the estimates differed between the two methods, the patterns of sedentary behaviour among groups (e.g., younger vs. older age groups, ethnic groups) were strikingly similar.

\section{Conclusions}

In conclusion, data from both subjective and objective sources demonstrate that sedentary behaviour accounts for a significant proportion of waking time and occurs across the full spectrum of behavioural contexts in NZ young people. In total, young people spent approximately 420-520 minutes per day being sedentary, comprised of $35 \%$ screen activities and $65 \%$ non-screen activities. The dual approach to measurement used here provides complementary data on not only the volume of sedentary time, but the context of what people are doing. The results highlight the potential for tailoring a sedentary behaviour intervention to a demographic group of interest, based on the pattern and popular types of sedentary behaviour in that group. In particular, males and older adolescents may benefit from reducing sedentary screen time, whereas females may benefit more by reducing non-screen sedentary behaviours. Those of Asian ethnicity may consider reducing total sedentary time. This study highlights the importance of considering all sedentary behaviours when designing interventions.

\section{Acknowledgements}

The sedentary behaviour analysis was supported by a grant from the Heart Foundation of New Zealand. The national survey was funded by Sport and Recreation New Zealand, the Ministry of Health, the Ministry of Education and the Ministry of Youth Development. LF was supported by a Tertiary Education Commission Bright Futures Doctoral Scholarship and is currently supported by a Heart Foundation of New Zealand Post-doctoral Fellowship. RM was supported by a Heart Foundation of New Zealand Fellowship. 


\section{Author details}

${ }^{1}$ Clinical Trials Research Unit, University of Auckland, Private Bag 92019, Auckland Mail Centre, Auckland 1142, New Zealand. ${ }^{2}$ Health and Use of Time (HUT) Group, Sansom Institute for Health Research, University of South Australia, GPO Box 2471, Adelaide, South Australia 5001, Australia. ${ }^{3}$ School of Education, Flinders University, GPO Box 2100, Adelaide, South Australia 5001, Australia.

\section{Authors' contributions}

LF was responsible for the conception and design of the sedentary behaviour analyses and was primarily responsible for drafting the manuscript. RM was responsible for the design of the original national survey. YJ undertook the survey analyses. All authors (LF, RM, YJ, TO and KR) contributed to the interpretation of data, critical revision of the manuscript and have given final approval of the manuscript.

\section{Competing interests}

The authors declare that they have no competing interests.

Received: 18 July 2011 Accepted: 1 December 2011

Published: 1 December 2011

\section{References}

1. Sardinha LB, Andersen LB, Anderssen SA, Quiterio AL, Ornelas R, Froberg K, Riddoch CJ, Ekelund U: Objectively measured time spent sedentary is associated with insulin resistance independent of overall and central body fat in 9- to 10-year-old portuguese children. Diabetes Care 2008, 31:569-575.

2. Steele RM, van Sluijs EMF, Cassidy A, Griffin SJ, Ekelund U: Targeting sedentary time or moderate- and vigorous-intensity activity: independent relations with adiposity in a population-based sample of 10-y-old British children. American Journal of Clinical Nutrition 2009, 90:1185-92.

3. Mitchell JA, Mattocks C, Ness AR, Leary SD, Pate RR, Dowda M, Blair SN, Riddoch C: Sedentary behavior and obesity in a large cohort of children. Obesity 2009, 17:1596-1602.

4. Chastin SFM, Granat MH: Methods for objective measure, quantification and analysis of sedentary behaviour and inactivity. Gait \& Posture 2010, 31:82-86.

5. Matthews CE, Chen KY, Freedson PS, Buchowski MS, Beech BM, Pate RR, Troiano RP: Amount of time spent in sedentary behaviors in the United States, 2003-2004. American Journal of Epidemiology 2008, 167(7):875-881.

6. Biddle $\mathrm{SJH}$ : Sedentary behavior. American Journal of Preventive Medicine 2007, 33(6):502-504.

7. Biddle SJ, Gorely T, Marshall SJ, Murdey I, Cameron N: Physical activity and sedentary behaviours in youth: issues and controversies. Journal of the Royal Society for the Promotion of Health 2004, 124(1):29-33.

8. Olds TS, Maher CA, Ridley K, Kittel D: Descriptive epidemiology of screen and non-screen sedentary time in adolescents. International Journal of Behavioral Nutrition and Physical Activity 2010, 7:92.

9. Sallis JF, Saelens BE: Assessment of physical activity by self-report: Status, limitations and future directions. Research Quarterly for Exercise and Sport 2000, 71:1-14.

10. Gorely T, Marshall SJ, Biddle SJH, Cameron N: The prevalence of leisure time sedentary behavior and physical activity in adolescent girls: An ecological momentary assessment approach. International Journal of Pediatric Obesity 2007, 2(4):227-234.

11. Treuth MS, Baggett CD, Pratt CA, Going SB, Elder JP, Charneco EY, Webber LS: A longitudinal study of sedentary behavior and overweight in adolescent girls. Obesity 2009, 17:1003-1008.

12. Healy GN, Clark BK, Winkler EAH, Gardiner PA, Brown WJ, Matthews CE: Measurement of Adults' Sedentary Time in Population-Based Studies. American Journal of Preventive Medicine 2011, 41(2):216-227.

13. Statistics New Zealand: 2006 Census Data - QuickStats About Culture and Identity (No.) Wellington: Statistics New Zealand; 2006.

14. ISAK: International Standards for Anthropometric Assessment (Trans. ed. Vol.) Canberra: ISAK; 2001

15. Cole TJ, Bellizzi MC, Flegal KM, Dietz WH: Establishing a standard definition for child overweight and obesity worldwide: international survey. British Medical Journal 2000, 320:1240-1243.
16. Ridley $\mathrm{K}$, Olds T, Hill A: The multimedia activity recall for children and adolescents (MARCA): development and evaluation. International Journal of Behavioral Nutrition and Physical Activity 2006, 3(10).

17. Ridley K, Ainsworth BE, Olds TS: Development of a compendium of energy expenditures for youth. International Journal of Behavioral Nutrition and Physical Activity 2008, 5:45.

18. Ainsworth BE, Haskell WL, Whitt MC, Irwin ML, Swartz AM, Strath SJ, O'Brien WL, Bassett DR, Schmitz KH, Emplaincourt PO, Jacobs DR, Leon AS: Compendium of physical activities: an update of activity codes and MET intensities. Medicine \& Science in Sports \& Exercise 2000, 32(9):s498-s516.

19. Jetté $M$, Sidney K, Blümchen G: Metabolic equivalents (METS) in exercise testing, exercise prescription, and evaluation of functional capacity. Clinical Cardiology 1990, 13(8):555-65.

20. Olds TS, Ridley K, Dollman J, Maher CA: The validity of a comptuerized use of time recall, the Multimedia Activity Recall for Children and Adolescents. Pediatric Exercise Science 2010, 22:34-43.

21. Rowlands AV: Accelerometer assessment of physical activity in children. Pediatric Exercise Science 2007, 19(3):252-266.

22. Treuth MS, Schmitz K, Catellier DJ, McMurray RG, Murray DM, Almeida MJ, Going S, Norman JE, Pate R: Defining accelerometer thresholds for activity intensities in adolescent girls. Medicine and Science in Sports and Exercise 2004, 36(7):1259-1266.

23. Trost SG, Ward DS, Burke JR: Validity of the Computer Science and Application (CSA) Activity Monitor in children. Medicine and Science in Sports and Exercise 1998, 30:629-633.

24. Treuth MS, Schmitz K, Catellier DJ, McMurray RG, Murray DM, Almeida MJ, Going S, Norman JE, R P: Defining accelerometer thresholds for activity intensities in adolescent girls. Medicine \& Science in Sports \& Exercise 2004, 36(7):1259-66.

25. Mâsse L, Fuemmeler BF, Anderson CB, Matthews CE, Trost S, Catellier DJ, Treuth MS: Accelerometer data reduction: A comparison of four reduction algorithms on select outcome variables. Medicine \& Science in Sports \& Exercise 2005, 37(11):S544-S554.

26. Salmond C, Crampton P, Atkinson J: NZDep2006 Index of Deprivation (No.) Wellington Department of Public Health University of Otago; 2007.

27. Ministry of Health: Presenting Ethnicity: Comparing prioritised and total response ethnicity in descriptive analyses of New Zealand Health Monitor surveys (No.) Wellington: Ministry of Health; 2008

28. Statistics New Zealand: Report of the review of the measurement of ethnicity (No.) Wellington: Statistics New Zealand; 2004.

29. Cole TJ, KM F, D N, AA J: Body mass index cut offs to define thinness in children and adolescents: international survey. British Medical Journal 2007, 335:194

30. Martínez-Gómez D, Welk GJ, Calle ME, Marcos A, Veiga OL: Preliminary evidence of physical activity levels measured by accelerometer in Spanish adolescents; The AFINOS Study. Nutricion Hospitalaria 2009, 24(2):226-232.

31. Gaya AR, Alves A, Aires L, Martins CL, Ribeiro JC, Mota J: Association between time spent in sedentary, moderate to vigorous physical activity, body mass index, cardiorespiratory fitness and blood pressure. Annals of Human Biology 2009, 36(4):379-387.

32. DeMattia $L$, Lemont $L$, Meurer $L$ : Do interventions to limit sedentary behaviours change behaviour and reduce childhood obesity? A critical review of the literature. Obesity Reviews 2007, 8:69-81.

33. Browne KD, Hamilton-Giachritsis C: The influence of violent media on children and adolescents:a public-health approach. Lancet 2005, 365(9460):702-10.

34. Rideout VJ, Foehr UG, Roberts DF: Generation M2: Media in the lives of 8- to 18-year-olds. A Kaiser Family Foundation Study (No.) Menlo Park California: Henry J. Kaiser Family Foundation; 2010.

35. Lanningham-Foster L, Foster RC, McCrady SK, Manohar CU, Jensen TB, Mitre NG, Hill JO, Levine JA: Changing the school environment to increase physical activity in children. Obesity 2008, 16(8):1849-53.

36. Healy GN, Dunstan DW, Salmon J, Cerin E, Shaw JE, Zimmet PZ, Owen N: Breaks in sedentary time: beneficial associations with metabolic risk. Diabetes Care 2008, 31(4):661-6.

37. Nader PR, Bradley RH, Houts RM, McRitchie SL, O'Brien M: Moderate-tovigorous physical activity from ages 9 to 15 years. Journal of the American Medical Association 2008, 300:295-305. 
38. Reilly JJ, Penpraze V, Hislop J, Davies G, Grant S, Paton JY: Objective measurement of physical activity and sedentary behaviour: review with new data. Archives of Disease in Childhood 2008, 93:614-619.

39. Healy GN, Anuradha S, Osman A, Masters J, Winkler E, Reeves M: Comparison of the GT3X-plus and activPAL monitors in controlled and free-living environments: accuracy and responsiveness to change. 2011 Annual Meeting of the International Society for Behavioral Nutrition and Physical Activity Melbourne, Australia; 2011.

doi:10.1186/1479-5868-8-132

Cite this article as: Foley et al:. It's not just the television: survey analysis of sedentary behaviour in New Zealand young people.

International Journal of Behavioral Nutrition and Physical Activity 2011 8:132.

Submit your next manuscript to BioMed Central and take full advantage of:

- Convenient online submission

- Thorough peer review

- No space constraints or color figure charges

- Immediate publication on acceptance

- Inclusion in PubMed, CAS, Scopus and Google Scholar

- Research which is freely available for redistribution

Submit your manuscript at www.biomedcentral.com/submit
C Biomed Central 